\title{
Long term follow-up after coronary artery bypass graft surgery Progression and regression of disease in native coronary
circulation and bypass grafts
}

\author{
R W BROWER, K LAIRD-MEETER, P W SERRUYS, G T MEESTER, P G HUGENHOLTZ \\ From the Interuniversity Cardiology Institute and Thoraxcenter, Erasmus University, Rotterdam, The Netherlands
}

SUMMARY Angiographically demonstrable changes in bypass status and their relation to the disease in the native coronary circulation were studied in 221 patients one year and three years after coronary artery bypass graft surgery. The extent of coronary artery disease was scored according to the recommendations of the American Heart Association and quantified following the method of Leaman. Patency in 570 grafts at one year was $79.6 \%$ and at three years $\mathbf{7 6 . 5 \%}$. The majority of grafts $(83.5 \%)$ showed no change from one year to three years, $11.4 \%$ showed progression in disease, and $5.1 \%$ showed regression. The majority of grafts which occlude do so in the first year after surgery. After the first year, the graft attrition rate is $1.6 \%$ of grafts per year.

The coronary score $(0$, no disease; $>30$, serious three vessel disease) before surgery was $14.2 \pm 1.92$ (mean $\pm 95 \%$ confidence) and dropped to $5 \cdot 3 \pm 0.76$ at one year when corrected for patent grafts. The coronary score remains greater than zero because of early graft closure and/or untreated lesions. By three years the corrected coronary score increased to $7.2 \pm 1.06$ primarily because of progression of disease in the native coronary circulation. Two subgroups, formed on the basis of angina pectoris at three years, showed that progression of disease in the native circulation was identical, but that return of angina was highly correlated with whether or not this disease occurred in segments perfused by patent grafts.

Those factors known to be risk factors for coronary artery disease do not appear to have a bearing on progression or regression of disease in the graft, nor does the extent of coronary artery disease at the time of surgery correlate with eventual graft patency.

There remains relatively little information on the progression and regression of disease in bypass grafts in the long term after coronary artery bypass graft surgery. This work was undertaken to document angiographically demonstrable changes in bypass status, the relation to the disease in the native coronary arteries, and the clinical factors possibly affected by this process.

This study was made possible by the existence of a large data bank designed for the three year follow-up of patients after bypass grafting. ${ }^{1}$ The pertinent aspects of this data bank are that it specifies recatheterisation at one year and three years after

Accepted for publication 10 March 1983 bypass surgery with complete scoring of the coronary angiogram and bypass graft status. A complete physical examination and history are also obtained at these points. The development in this laboratory of a coronary scoring system to quantify the extent of coronary artery disease and its changes over the follow-up period has been previously reported. ${ }^{2}$ This method is also applied here in this study.

\section{Methods}

The selection criteria for this study was the availability in Rotterdam of selective graft and coronary angiograms at one year and three years after coronary artery bypass graft surgery. All patients were also evaluated before surgery. In total 221 patients met 
these criteria. All were adults, mean age 52 years, and $96 \%$ were men. On the basis of clinically available information on medical treatment and functional class, these patients did not differ from those refusing recatheterisation but seen in the out-patient clinic as part of the routine follow-up after coronary artery bypass grafting.

The purpose of the coronary score, developed by Leaman et al., ${ }^{2}$ was to quantify the severity of underlying coronary artery disease before surgery, and the effectively untreated disease remaining after coronary artery bypass grafting. The different coronary vessels carry different volumes of blood to the left ventricle, and the coronary score was derived to take this into account.

The left coronary artery carries approximately five times the amount of flow to the left ventricle compared with the right coronary artery. ${ }^{3-5}$ It has also been shown that in the usual right dominant coronary system, the flow in the left coronary artery is $66 \%$ into the left anterior descending and $33 \%$ into the circumflex coronary artery. ${ }^{3}$ Thus, the left anterior descending carries approximately three and a half times and the circumflex one and a half times as much blood as the right coronary artery. This then was the basis for assigning different weighting values to the different coronary arteries. The coronary arteries were subdivided into segments according to the scheme devised by the American Heart Association. ${ }^{6}$ The right coronary artery does not supply blood to the left ventricle until the posterior descending branch is given off, so only the segment with the most severe disease was analysed. The left anterior descending and circumflex coronary arteries, however, were further subdivided and weighting factors were assigned to each segment (Fig.1). As shown in the figure, different weighting factors were assigned in the event of left dominance.

The degree of stenosis was also considered in deriving the coronary score. If the vessel was totally occluded, the coronary artery segment value was multiplied by 5.0 . If there was a 90 to $99 \%$ occlusion (luminal diameter narrowing), it was multiplied by 3.0. For a 50 to $90 \%$ obstruction, it was multiplied by 1.0. The segmental scores were then added to derive the total coronary score. A score of zero would indicate no obstructive coronary artery disease, and the higher the score, the greater the obstruction. The role of coronary collaterals has been previously studied and found not to effect the outcome of surgery. ${ }^{7}$

In deriving the postoperative coronary score, a vessel segment was considered normal if it was supplied by a patent aortocoronary bypass graft that was free of occlusive disease. If the aortocoronary bypass graft was occluded, the score was derived from the degree of disease in the native coronary system; that is, the
Extent of narrowing

(coronary disease factor)

$$
\begin{array}{r}
50-89 \%: 1 \\
90-99 \%: 3 \\
100 \%: 5
\end{array}
$$$$
\text { Significance of vessel }
$$$$
\text { ( vessel factor) }
$$

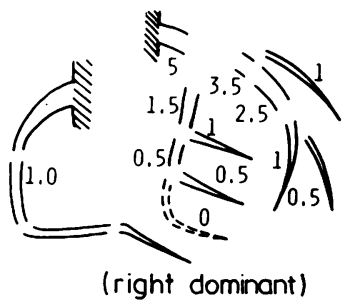

$$
C S=\sum_{15}^{1} \begin{aligned}
& \text { coronary disease } X \begin{array}{l}
\text { vessel } \\
\text { factor }
\end{array} \\
& \text { Range } 0 \text { (no CAD) to } \angle 0 \text { (serious CAD) }
\end{aligned}
$$

Fig. 1 The coronary score was formulated to give a single number representing the severity of coronary artery disease as it affects perfusion to the left ventricle (Leaman et al. ${ }^{2}$ ) See text for detailed description. A value of zero represents no coromary artery disease, while 40 would represent very serious three vessel disease.

score was derived as if no graft had been placed. Hypertension was defined as a diastolic arterial pressure in excess of $95 \mathrm{~mm} \mathrm{Hg}$.

Statistical analysis included the Student's paired t test, $\chi^{2}$, or analysis of variance where appropriate. The lower limit for significance was taken as $\mathbf{p}=\mathbf{0 . 0 5}$, two sided distribution. Unless otherwise stated, data are presented as the mean \pm the $95 \%$ confidence interval of the mean.

\section{Results}

There were 221 patients who met the inclusion criteria of angiography performed at one year and three years after surgery according to the study procedure.

The ejection fraction before operation was $0.60 \pm 0.12$ (mean $\pm S D$ ), at 1 year it was $0.60 \pm 0.13$, and at 3 years it was $0.59 \pm 0.12$. There was thus no clinically important change in global left ventricular function for the group as a whole.

The coronary score (native and corrected for grafting) is summarised in Table 1 , and for the sake of clarity illustrated in Fig. 2. At operation the mean coronary score for the entire series was $14.2 \pm 1.02$ (mean $\pm 95 \%$ confidence interval for the mean). One year after operation the corrected coronary score fell to $5 \cdot 3 \pm 0.76$. This is not zero because some grafts have already occluded, and not all disease could be effectively bypassed.

From one year to three years there was some progression on average in the corrected coronary score (from 5.3 to $7 \cdot 1$ ). During this period the uncorrected 
Table 1 Coronary score before surgery and one and three years after surgery in all 221 patients, group 1 (126 patients, no evidence of angina pectoris at three years), and group 2 (95 patients, angina pectoris present at three years)

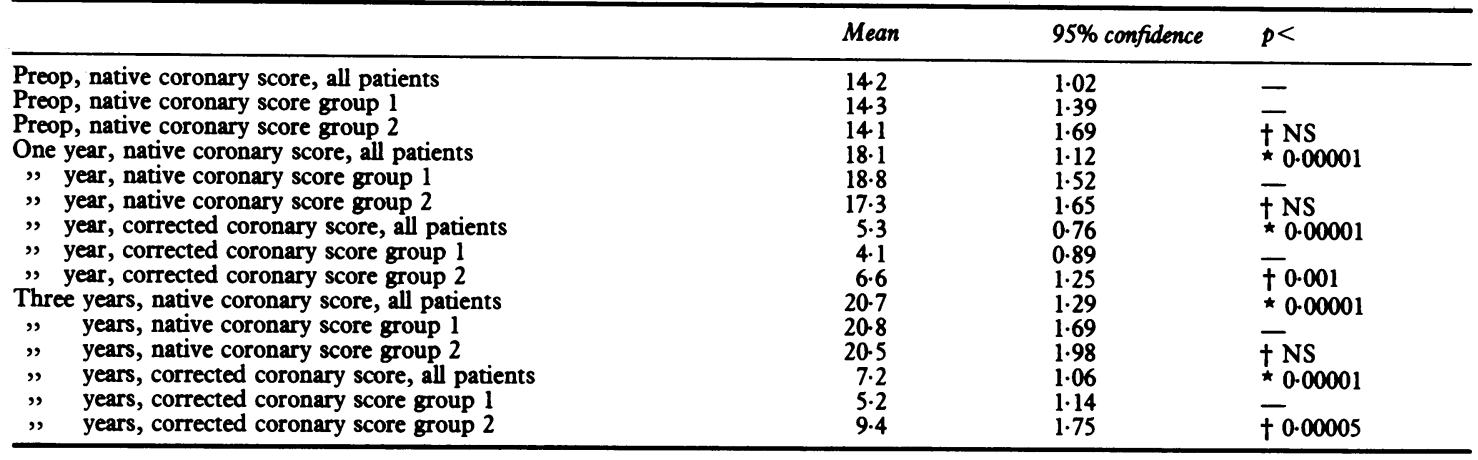

Native coronary score is shown as well as that corrected for patent grafts. Data presented as the mean $\pm 95 \%$ confidence interval. Abbreviations: *comparison of difference with respect to preop coronary scores (Student's paired t test); tstatistically significant difference between groups 1 and 2 (Student's unpaired t test); NS no significant difference

coronary score in the native circulation increased from $18 \cdot 1$ to $20 \cdot 7$ ( $16 \%$ increase).

The graft status is summarised in Table 2. There were $\mathbf{5 7 0}$ grafts scored, giving a revascularisation rate of 2.6 grafts per patient. The patency rate at one year was $79.6 \%$ and at three years $76.5 \%$. That is, $3.1 \%$ more grafts occluded during the two year interval between follow-ups, giving a graft loss rate of $1.6 \%$ of grafts per year after the first year. During the followup period the majority of grafts $(83.5 \%)$ showed no change within the scoring limits used in this study, but $11.4 \%$ of grafts were coded as progressing in severity of stenosis by one or more classes while $5 \cdot 1 \%$ were classed as regressing by one or more classes. As the corrected coronary score was computed based on patency vs occlusion of the graft (see methods) the progression in the corrected coronary score is the

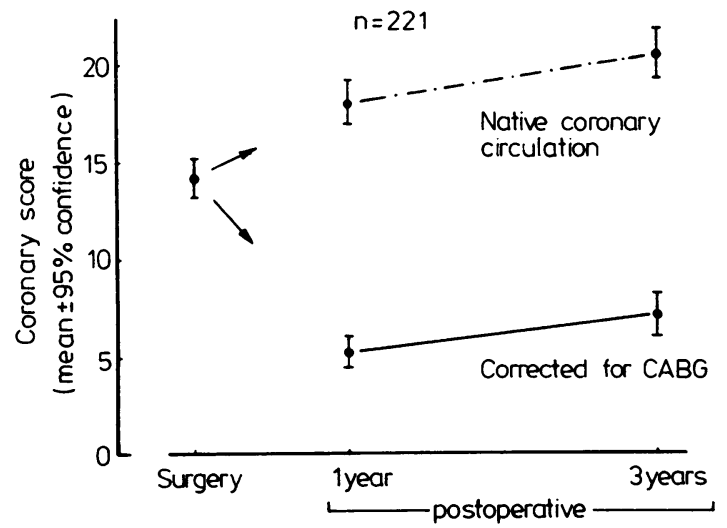

Fig. 2 The coronary score in the native circulation and corrected for bypass grafting before surgery, and one and three years afterwards. Vertical bars represent the mean $\pm 95 \%$ confidence limits for the mean in 221 patients. result primarily of progression of disease in the native coronary circulation.

The relation between recurrence of angina pectoris and progression of disease was also studied. At three years after operation $43 \%$ of patients reported they experienced some angina, though usually of less severity (ease of provocation and frequency of attacks) than previously.

Two groups were formed on the basis of angina pectoris at three years (group 1: no evidence of angina pectoris, and group 2: presence of angina pectoris at three years), and the coronary score was compared for all follow-ups (Table 1).

The preoperative coronary scores for groups 1 and 2 were, respectively, $14.3 \pm 1.39$ (mean $\pm 95 \%$ confidence interval) and $14 \cdot 1 \pm 1.57$ (no significant difference). That is, there were no differences in

Table 2 Summary of graft status at one year and three years after surgery

\begin{tabular}{|c|c|c|c|c|c|}
\hline & \multicolumn{5}{|c|}{3 year follow-up } \\
\hline ear & Normal & $50 \%$ & $50-90 \%$ & $91-99 \%$ & $100 \%$ \\
\hline Normal & 309 & 31 & 5 & 1 & 10 \\
\hline $50 \%$ & 15 & $34-$ & 6 & 1 & 4 \\
\hline $51-90 \%$ & 3 & 8 & 18 & 1 & 2 \\
\hline $91-99 \%$ & 0 & 1 & 1 & 1 & 4 \\
\hline $100 \%$ & 0 & 0 & 1 & 0 & -114 \\
\hline \multicolumn{6}{|c|}{$\begin{array}{l}\text { Total number of grafts: } 570 \\
\text { progression: } 65114 \%\end{array}$} \\
\hline $\begin{array}{l}\text { Stability } \\
\text { regressic }\end{array}$ & $\begin{array}{c}476 \quad 83.5 \% \\
29 \\
29.1 \%\end{array}$ & & & & \\
\hline
\end{tabular}

Five classes of occlusion are shown: $100 \%$ occlusion, 91 to $99 \%$ occlusion, 50 to $90 \%$ occlusion, less that $50 \%$ obstruction, and normal graf. Entries on the diagonal represent stability in graft status from one year to three years. Entries above the diagonal represent progression of disease, while entries below the diagonal represent regression. 
Table 3 Summary of risk factors, associated with coronary artery disease, but studied in relation to progression, stability, or regression of disease in grafi ( $n=570$ grafts, see Table 2) from one year to three years; analysis of variance of $\chi^{2}$ contingency table used for statistical analysis

\begin{tabular}{|c|c|c|c|c|}
\hline & Progression & Stable & Regression & $p$ \\
\hline $\begin{array}{c}\text { Cholesterol }(\mathrm{mmol} / \mathrm{l}) \\
(\text { mean } \pm \mathrm{SD})\end{array}$ & $\begin{array}{l}(7 \cdot 88 \pm 2 \cdot 01) \\
n=52\end{array}$ & $\begin{array}{l}(7.60 \pm 2.03) \\
\mathrm{n}=353\end{array}$ & $\begin{array}{l}(8.42 \pm 2.0) \\
\mathrm{n}=23\end{array}$ & NS \\
\hline $\begin{array}{l}\text { (yes or no) } \\
\text { Use of anticoagulants }\end{array}$ & $y=30 n=35$ & $y=206 n=270$ & $y=12 n=17$ & NS \\
\hline $\begin{array}{l}\text { (yes or no) } \\
\text { Hypertension }\end{array}$ & $y=29 n=36$ & $y=193 n=279$ & $y=17 n=12$ & NS \\
\hline $\begin{array}{l}\text { (yes or no) } \\
\text { Preop coronary score }\end{array}$ & $y=22 n=43$ & $y=154 n=322$ & $y=12 n=17$ & NS \\
\hline$($ mean \pm SD) & $145 \pm 8.0$ & $16 \cdot 1 \pm 7 \cdot 8$ & $12 \cdot 0 \pm 7 \cdot 7$ & NS \\
\hline
\end{tabular}

coronary score between the two groups at the outset. At one year the uncorrected (native) coronary score was $18.8 \pm 1.52$ and $17.3 \pm 1.65$ (no significant difference), while the corrected score was $4.1 \pm 0.89$ and $6.6 \pm 1.25(\mathrm{p}<0.001)$. At three years, this trend is even more apparent; the uncorrected coronary score was $20.8 \pm 1.69$ and $20.5 \pm 1.98$ (no significant difference), while the corrected coronary score was $5.2 \pm 1.14$ and $9.4 \pm 1.75(p<0.00005)$. Thus, in those patients reporting angina pectoris at three years, the coronary score (corrected for patent grafts) shows a clear trend towards preoperative values while those patients without angina pectoris showed minimal progression in the corrected coronary score. This occurred in spite of the fact that the coronary score for the native circulation (uncorrected) was virtually identical in the two groups. It appears that progression of disease in the coronary arteries is present to a similar extent in both groups, but group 1 (without angina pectoris) had this disease more effectively bypassed than group 2 patients.

The relation between risk factors for coronary artery disease and graft status from one year to three years after surgery is summarised in Table 3 . The first entry gives the mean and standard deviation of cholesterol for the three classes of bypass grafts: those where there was progression of disease, stability, and regression of disease. Results for smoking, use of anticoagulants, presence of hypertension, and preoperative coronary score are also summarised in Table 3. There were no significant correlations found for any of these risk factors.

\section{Discusssion}

There are a number of limitations to this study which should be pointed out at the outset. The analysis obviously excludes those patients who died within three years of operation and this could bias any conclusions based on the surviving patients. To place this factor in perspective, the mortality for uncomplicated bypass grafting over the period of this study was $2 \cdot 1 \%$ in the first year and an additional $1.9 \%$ from one year to three years. Therefore, this source of bias is not large, but would tend in any event towards one being overly optimistic concerning progression of disease. Secondly, in deriving the corrected score after bypass surgery the grafts were characterised as either patent or occluded. As shown in Table 2, this is an oversimplification necessitated by the fact that there is no accurate way of determining whether the combined disease in a patent graft and coronary artery is effectively limiting blood supply except in the extreme when the graft is occluded.

The magnitude of this problem can be estimated from Table 2 . At one year $79.6 \%$ of grafts were classed as "patent"; this includes $7 \%$ of the total which had disease in the grafts scored between 51 and $99 \%$. At three years, $6 \%$ of grafts were so scored in spite of more grafts becoming occluded. Thus, while this system of classifying grafts as either patent or occluded is an oversimplification, it would not appear to bias the study as far as progression of disease is concerned.

It could be argued that progression/regression of disease is sufficiently gradual that only small changes are likely to occur between the one and three year follow-up studies and that the American Heart Association recommendations for scoring coronary angiograms (five disease classes, 15 segments) 6 may not be sufficient. There is a limit, related to the inter- and intra-observer variability, to which small changes can be reliably scored. Reports of observer variability provide some guidelines, ${ }^{8-10}$ but the analysis has not been taken to its logical conclusion, that is development of an optimal scoring system commensurate with the "signal to noise ratio" of the diagnostic technique and experienced observer.

The above cited reports all employ a coronary map of about the same number of segments as we used, but most use only two or three classes of disease compared with our five. For example, Sanmarco et al.$^{8}$ report a 95\% consistency in an expert panel when scoring the presence or absence of a significant lesion $(>70 \%$ area 
stenosis or $>50 \%$ diameter stenosis). Only two classes clearly provide a very limited resolution for studies of progression of disease, but could still be useful. Whether two or five classes are used, it is essential to keep these criteria in mind when evaluating changes in disease status.

Bourassa et al. ${ }^{11}$ have reported that there is an accelerated progression of disease in proximal segments of grafted coronary arteries occurring primarily in the first year, but then remaining relatively stable afterwards. Natural progression of disease in non-grafted arteries continues over the long term though possibly in bouts. ${ }^{12}$ The progression in the corrected coronary score from one to three years therefore primarily reflects the development of disease in non-grafted segments, and to a lesser extent the consequences of graft occlusion. Nevertheless, between one and three years, we do find some progression of disease in segments either directly or indirectly bypassed. Providing that the graft remains open, this progression is not associated with the return of angina pectoris in contrast to the situation where progression of disease occurs in arteries not bypassed.

The data summarised in Fig. 2 lead to several speculations. For example, if the dashed line connecting the one year and three year native coronary score is extrapolated to the time of operation, it projects a coronary score 2.6 units above that obtained at the time. This may be ascribed to damage to the coronary arteries as a result of the operation itself or indirectly by the establishment of new perfusion pathways. It is also apparent that the line connecting the corrected coronary score could be extended into the future where it would eventually intersect the level of the coronary score before surgery. While little confidence can be attached to such a long term extrapolation, this point can be calculated to be about 10 years after operation. This estimate is probably too pessimistic as the majority of disease progression is known to occur early in the follow-up.

It was found that a minority of grafts $(5 \cdot 1 \%)$ show some regression in disease. In view of the above mentioned observer variability in scoring angiograms, it can be debated whether this figure represents true regression as opposed to observer variability. Studies conducted in our laboratory suggest that an observer variability of $5 \%$ is not unreasonable, and that the entire series showing regression could be ascribed to observer variability. Therefore, all these angiograms were reviewed at least twice, and in our opinion these results do reflect actual regression of disease. Bruschke et al. ${ }^{13}$ have also reported regression of disease in $4.7 \%$ of patients not operated upon.

No significant correlations were found between the conventional risk factors and progression of disease in the grafts (Table 3). This also appears to be the case for progression of disease in coronary arteries. ${ }^{12-15}$ Allard et al., ${ }^{16}$ however, have reported that hypertriglyceridaemia, a factor which we did not examine, is associated with a higher risk of graft occlusion. It is particularly interesting to note that the preoperative coronary score had no bearing on disease in the bypass graft. This most clearly indicates the decoupling of conventional risk factors in coronary artery disease with later development of disease in the graft. On reflection, this approach to the problem is an oversimplification. These risk factors for a patient are equally present for all the patient's grafts, yet it is a common observation that only one graft will show progression of disease while the others are stable or vice versa. The data presented in Table 3 therefore only confirm that the conventional wisdom of risk factors in coronary artery disease does not apply to the graft itself. Other factors, including intimal hyperplasia which is not well understood, are playing a more decisive role.

Several conclusions are apparent. The majority of bypass grafts which occlude do so in the first year after bypass grafting. Afterwards, there is a much slower graft attrition rate, but one which if continued at a steady rate would result in half of all grafts becoming occluded in 15 years. A far more significant factor appears to be the progression of disease in the native circulation, part of which is bypassed and part of which is not. In the latter case, there is a clear connection between the return of angina pectoris at three years and progression of coronary artery disease. Between one year and three years after coronary artery bypass surgery we find that most grafts are stable, there is progression of disease in $11 \%$, and regression in 5\%. Regression of disease in the graft is infrequent, but can occasionally be shown. Those factors known to be risk factors for coronary artery disease do not appear to have a bearing on progression or regression of disease in the graft.

The authors acknowledge the assistance of $\mathrm{H} \mathrm{J}$ ten Katen in preparing the drawings and performing the data bank searches for this study. This study was supported as project $4 \mathrm{c}$ from the Interuniversity Cardiology Institute.

\section{References}

1 Brower RW, ten Katen HJ, Meester GT. Interim data processing in the Netherlands study on coronary artery bypass graft surgery. Comput Biomed Res 1980; 13: 87101.

2 Leaman DM, Brower RW, Meester GT, Serruys P, vd Brand M. Coronary artery atherosclerosis: severity of the disease, severity of angina pectoris, and compromised left ventricular function. Circulation 1981; 63: 285-92. 
3 Kalbfleisch H, Hort W. Quantitative study on the size of coronary artery supplying areas postmortem. Am Heart $\mathcal{f}$ 1977; 94: 183-8.

4 Dwyer EM, Dell RB, Cannon PJ. Regional myocardial blood flow in patients with residual anterior and inferior transmural infarction. Circulation 1973; 48: 924-35.

5 Ross RS, Ueda K, Lichtlen PR, Rees JR. Measurement of myocardial blood flow in animals and man by selective injection of radioactive inert gas into the coronary arteries. Circ Res 1964; 15: 28-41.

6 American Heart Association, Council on Cardiovascular Surgery. Report of the Ad Hoc committee for Grading of Coronary Artery Disease: a reporting system on patients evaluated for coronary artery disease. Dallas: American Heart Association, document 73-315-A. (Published in Circulation 1975; 51: 7-40.)

7 Brower RW, ten Katen HJ, vd Brand M. Do coronary collaterals influence the outcome of bypass graft surgery. Thorac Cardiovasc Surg 1982; 30: 259-64.

8 Sanmarco ME, Brooks SH, Blankenhorn DH. Reproducibility of a consensus panel in the interpretation of coronary angiograms. Am Heart $\mathcal{f}$ 1978; 96: 430-7.

9 Zir LM, Miller SW, Dinsmore RE, Gilbert JP, Harthorne JW. Interobserver variability in coronary angiography. Circulation 1976; 53: 627-32.

10 Detre KM, Wright E, Murphy ML, Takaro T. Observer agreement in evaluating coronary angiograms. Circulation 1975; 52: 979-86.

11 Bourassa MG, Lespérance J, Corbara F, Saltiel J, Campeau L. Progression of obstructive coronary artery disease 5 to 7 years after aortacoronary bypass surgery. Circulation 1978; 58 (suppl I): I-100-6.

12 Kimbiris D, Segal BL. Coronary disease progression in patients with and without saphenous vein bypass surgery. Am Heart $\mathcal{F}$ 1981; 102: 811-8.

13 Bruschke AVG, Wijers TS, Kolsters W, Landmann J. The anatomic evolution of coronary artery disease demonstrated by coronary arteriography in 256 nonoperated patients. Circulation 1981; 63: 527-36.

14 Bemis CE, Gorlin R, Kemp HG, Herman MV. Progression of coronary artery disease. A clinical arteriographic study. Circulation 1973; 47: 455-64.

15 Kramer JR, Matsuda Y, Mulligan JC, Aronow M, Proudfit WL. Progression of coronary atherosclerosis. Circulation 1981; 63: 519-26.

16 Allard C, Goulet C, Grondin CM, Lespérance J, Bourassa MG. Patency of aorta coronary vein grafts and serum triglycerides. Am $\mathcal{F}$ Cardiol 1974; 33: 679-80.

Requests for reprints to $\mathrm{Dr} R \mathrm{~W}$ Brower, Ee 2332, Erasmus University, POB 1738, 3000 DR Rotterdam, The Netherlands. 